\title{
NIVELES DE AUDIOMETRÍA Y SU RELACIÓN CON EL RUIDO OCUPACIONALEN ESTUDIA NTES DE LA ESCUELA PROFESIONAL DE ODONTOLOGÍA DE LA UNIVERSIDAD NACIONAL JORGE BASADRE GROHMANN EN TACNA-2016
}

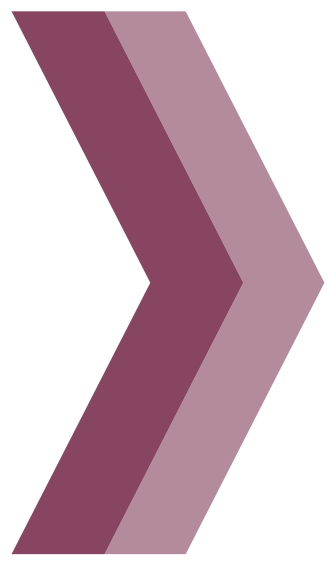

LEVELS OF AUDIOMETRY AND $\begin{array}{lllllllllllll} & \boldsymbol{T} & \boldsymbol{S} & \mathbf{R} & \mathbf{E} & \mathbf{L} & \mathbf{A} & \mathbf{T} & \boldsymbol{I} & \mathbf{O} & \mathbf{N} & \mathbf{T} & \mathbf{O}\end{array}$ OCCUPATIONAL NOISE IN $S$ T U D E N T S O F T H E PROFESSIONAL SCHOOL OF DENTISTRY AT JORGE BASADRE GROHMA N N NATIONAL UNIVERSITY, TACNA-2016

\section{Noelia Yesica Martinez Cántaro' Isabel Ayca Castro" Wender Condori Quispe ${ }^{3}$}

1. Cirujano Dentista. Maestro en Odontología con mención en Patología. Docente de la Escuela Profesional de Odontología de UNJBG.

2. Cirujano Dentista. Maestro en Odontoestomatología. Especialista en Odontopediatría. Docente de la Escuela Profesional de Odontología de UNJBG.

3. Cirujano Dentista. Especialista en Ortodoncia. Docente de la Escuela Profesional de Odontología de UNJBG.

\section{ABSTRACT}

INTRODUCTION: The dental practice is exposed to high noise from clinical training, which can be potentially harmful; since it affects the quality of life, causing acceleration of the hearing loss that is irreversible, but preventable. The objective of this study is to determine if there was a relationship between the levels of audiometry and the occupational noise in the students of the School of Dentistry at Jorge Basadre Grohmann National University in Tacna-Peru. MATERIALS AND METHODS: A descriptive-correlational crosssectional study was carried out with a total of 74 students. The participants were randomly selected, and they agreed to participate through informed consent. A survey and audiometric tests were then applied by a duly certified otolaryngologist. In addition, the noise level in decibels produced in clinical and laboratory practices was recorded with a standardized sound level meter. 
RESULTADOS: Se determinó que existe relación de los niveles de audiometría y ruido ocupacional $(p<0,050)$. El $62 \%$ de los estudiantes presentó audiometría normal, estando dentro del límite permisible (DLP), un $43.2 \%$ de los alumnos presentó hipoacusia ligera, estando dentro del límite permisible (DLP). Además, se observó que los niveles de ruido ocupacional DLP es de mayor porcentaje en el primer año; mientras que el mayor porcentaje de ruido ocupacional fuera del límite permisible (FLP) estuvo en el IV y $\mathrm{V}$ año. Los niveles audiométricos registraron un $48,6 \%$ como audición normal, mientras que el menor porcentaje $(14,9 \%)$ como hipoacusia leve. Se constató que los estudiantes con más años de exposición a ruido odontológico presentaron peor desempeño en las pruebas audiométricas. CONCLUSIÓN: Existe relación significativa entre los niveles de audiometría y ruido ocupacional en los estudiantes de la escuela de odontología en la UNJBG.

Palabras claves: Ruido ocupacional, audiometría, estudiantes de odontología.
RESULTS: It was determined that there is a relationship between the levels of audiometry and occupational noise $(p<0.050) .62 \%$ of the students presented normal audiometry, being within the permissible limit (DLP), $43.2 \%$ of the students had slight hearing loss, being within the permissible limit (DLP). In addition, it was observed that the levels of occupational noise DLP was of greater percentage in the first year; while the highest percentage of occupational noise outside the permissible limit (FLP) was present in the IV and V year. The audiometric levels recorded $48.6 \%$ as normal hearing, while the lowest percentage (14.9\%), as mild hearing loss. It was found that students with more years of exposure to dental noise had worse performance in audiometric tests. CONCLUSION: There was a significant relationship between the levels of audiometry and occupational noise in the students of the dental school at the UNJBG.

Keywords: Occupational noise, audiometry, dental students.

\section{INTRODUCCIÓN}

El ruido ha sido definido como la percepción indeseada. Es un sonido molesto para el oído, el cual produce efectos adversos fisiológicos o psicológicos, lo que interfieren con las actividades humanas de comunicación, trabajo y descanso. La intensidad del sonido y el tiempo de exposición son factores importantes al momento de determinar que tanto se puede ver afectada la capacidad auditiva de quienes están expuestos a determinados sonidos (1). El ruido ocupacional se genera en condiciones laborales, y afecta a millones de trabajadores en el mundo (2). Este tipo de ruido es la segunda causa más común de pérdida auditiva neurosensorial (3).

Cuando el oído se ve expuesto a ruido traumático no sólo se afectan las células ciliadas, sino también la organización del órgano de corti y su función (4). Si el sonido es lo suficientemente intenso, además de producirse una alteración física de la cóclea, otras estructuras también pueden ser dañadas, tales como la estría vascular y las células de soporte. Algún tiempo después de la muerte de células ciliadas también se puede producir un daño a nivel neural (4). El oído humano es capaz de percibir y soportar sonidos correspondientes a niveles de presión sonora entre 0 y 120 dB. Este último nivel de ruido marca aproximadamente el denominado "umbral del dolor". A niveles de ruido superiores pueden producirse daños físicos como rotura del tímpano (5). El ruido es causal importante de la contaminación por exceso o continuidad que puede afectar al propio oído así como también al sistema nervioso (6).

Una visión holística del lugar de trabajo, considera una combinación de factores físicos, químicos, biológicos y elementos que se relacionan con la salud y el bienestar de los trabajadores. Basado en este enfoque, se han generado iniciativas para investigar los efectos combinados de la exposición laboral al ruido y otros factores nocivos en la audición. En particular, es la interacción potencial (y posiblemente sinérgica) entre el ruido y las sustancias químicas, lo que se plantea como un nuevo reto para los investigadores del área (7).

En las clases prácticas de odontología, el medio ambiente acústico se caracteriza por altos niveles de ruido en comparación con otras áreas de enseñanza. Esto se debe al ruido que producen algunos instrumentos dentales y, además, al uso de los equipos 
por muchos usuarios a la vez. Esta situación se agrava cuando las salas de clases tienen superficies duras que actúan como reflectores de ruido, lo cual es la práctica más frecuente (8).

Estudios realizados han demostrado que la exposición constante a altos niveles de ruido no sólo trae como consecuencia la pérdida auditiva, sino que también reduce la capacidad de concentración. Por tanto, el costo de realización de una actividad específica se ve incrementado. A su vez, se predispone al trabajador a un estado más "irritable" luego de la actividad laboral, impidiendo un descanso y recuperación adecuado (9). La pérdida de la audición implica el cambio en el umbral auditivo detectado mediante audiometría (10).

La hipoacusia ocupacional afecta principalmente la capacidad del individuo para interactuar tanto en el trabajo como en lo social. Estas dificultades comunicacionales pueden llevar al individuo al aislamiento social, lo que impacta directamente en su calidad de vida (11).

Obando y cols. (12) realizaron un estudio descriptivo para conocer las características auditivas en auxiliares de odontología y odontólogos entre los 20 - 50 años de edad, a quienes se les aplicó una evaluación audiológica completa, concluyendo que el $22 \%$ de la población presentó pérdida de la sensibilidad auditiva y con respecto al procesamiento auditivo de la información, entre el 10 y el $30 \%$ de la población presentó dificultad en las diferentes pruebas.

Sampaio y cols. (8) estudiaron los niveles de ruido en escuelas dentales. Los niveles sonoros fueron medidos en cinco diferentes áreas de práctica y laboratorios, seleccionados en dentro de una variedad de actividades de enseñanza-aprendizaje. Los autores hallaron que los niveles de ruido detectados en este estudio se consideran cerca del límite de riesgo de pérdida de la audición.

Fuentes y cols. (13) realizaron un estudio descriptivo y comparativo entre un grupo expuesto a ruido ocupacional y otro no. El grupo expuesto contaba con 50 estudiantes de la carrera de odontología que poseían al menos un semestre de exposición a ruido generado por los instrumentos de trabajo dental. Por otro lado, el grupo no expuesto contaba con 107 estudiantes pertenecientes a otras carreras. Los autores concluyeron que no había diferencia estadísticamente significativa al comparar los umbrales de ambos grupos tanto para el oído derecho como para el izquierdo.

Grass Martínez y cols. (14) realizaron un estudio observacional, descriptivo y transversal de 59 trabajadores que se encontraban laborando físicamente en el Servicio de Estomatología del Policlínico Docente "Julián Grimau García" de Santiago de Cuba, desde julio de 2015 hasta igual mes de 2016. El estudio tuvo como objetivo identificar los niveles de ruido existentes en el ambiente laboral de dicho servicio. En la serie se obtuvo que los departamentos de mayor nivel de ruido resultaron ser: Prótesis con 73,2 dB, Ortodoncia y Periodoncia con 72,7 dB, y Conservadora con 71,2 dB, en ese orden. Asimismo, en cuanto al ruido de fondo, las mayores mediciones se registraron en los departamentos de Conservadora y de Prótesis con 68,6 y 68,1 dB, respectivamente. Por otra parte, $62,7 \%$ del total de trabajadores presentaron problemas de hipoacusia por estar expuestos permanentemente a este agente físico negativo.

Paredes G. (15) hizo un estudio que buscó determinar la relación entre el nivel de audición y el ruido ocupacional en el personal odontológico. Para tal efecto, 82 individuos fueron evaluados a través de una encuesta, entre ellos 36 odontólogos, 14 internos y 32 asistentes dentales del departamento de Estomatología del Centro Médico Naval Cirujano Mayor Santiago Távara "CMST". Seguidamente, se encontró que el $40 \%$ de la población presentó hipoacusia neurosensorial y trauma acústico; el ruido fuera del límite permisible medido en los consultorios representaba el 72\%. El 100\% de la población no usaba protección acústica mientras trabajaba. Se determinó que existe relación entre el ruido ocupacional y el nivel de audición en el personal odontológico. Se observó también que existe relación entre el trauma acústico e hipoacusia en el personal odontológico. Adicionalmente, se encontró correlación entre la presencia de trauma acústico y los años de servicio clínico y diferencia entre la presencia de hipoacusia y el número de años de servicio.

Finalmente, un estudio realizado a estudiantes de odontología de México en el año 2012 concluyó que es importante implementar los cuidados preventivos de la salud ocupacional desde los primeros años en clínicas, usando los tapones para mitigación de ruido como protocolo de seguridad laboral (16).

Por lo expuesto anteriormente, el conocimiento de la afectación del ruido ocupacional en los estudiantes de 
odontología se presenta como fundamental para poder evaluar medidas a fin de prevenir e implementar de herramientas útiles que permitan el mejoramiento y la optimización del entorno en el cual se desarrollan tanto académico como social y del servicio odontológico en la clínica de la Escuela de Odontología de la UNJBG.

\section{MATERIALES Y MÉTODOS}

El presente trabajo de investigación es un estudio descriptivo, correlacional de corte transversal y clínico. El universo estuvo conformado por 182 estudiantes de la Escuela Profesional de Odontología de la UNJBG. Para la selección de la muestra, se utilizó el método de muestreo estratificado con afijación proporcional por facultades, mientras que para la selección de la unidad de análisis se consideró el muestreo aleatorio simple (MAS). La muestra fue de 74 estudiantes siendo 18 de primer año, 14 del segundo año, 13 del tercer año, 19 del cuarto año y 10 del quinto año.

De esta forma, fueron incluidos los estudiantes del primer al quinto año de estudios de la Escuela Profesional de Odontología, matriculados durante el año académico 2016. Es importante precisar que los alumnos recibieron y aceptaron el consentimiento informado; y que fueron excluidos los estudiantes que presentaron enfermedades o infecciones auditivas.

A fin de recaudar datos, se utilizó la técnica de análisis documental debidamente validado. Asimismo, se aplicó una encuesta de conductas auditivas y ficha audiométrica, las que fueron ejecutadas por un profesional otorrinolaringólogo debidamente certificado. Además, se registró el nivel de ruido en decibeles producido en las prácticas clínicas y de laboratorio con un sonómetro estandarizado. El software SPSS v20 y la hoja de cálculo Excel fueron utilizados para el procesamiento de datos.

Para la prueba de la hipótesis se usó la prueba t de Student con un valor de significancia de $<0,05$. El análisis relacional se realizó con la prueba de chi cuadrado, el cual describe la asociación de las variables ruido ocupacional y nivel de audiometría.

\section{RESULTADOS}

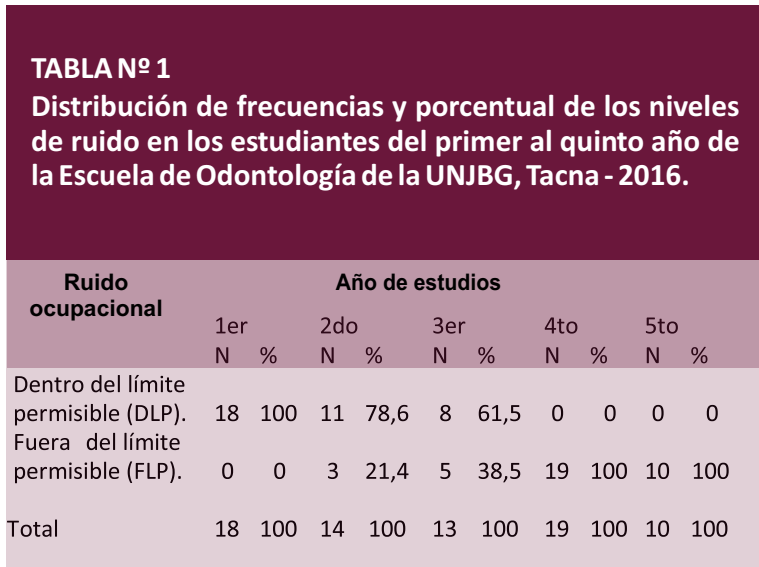

Fuente: Elaboración propia.

Según la tabla, se observa que el $100 \%$ de los estudiantes del primer año estuvo expuesto al ruido ocupacional DLP. Por otro lado, se observa que el $100 \%$ de los estudiantes del cuarto y quinto año estuvo expuesto a ruido ocupacional FLP. Esto evidencia que el ruido ocupacional aumentó en los últimos años de estudio.

\section{TABLA No 2}

Distribución de frecuencias y porcentual de los niveles de audiometría en los estudiantes de la Escuela de Odontología de la UNJBG.

\begin{tabular}{lcc}
\hline & & \\
Audiometría & Frecuencia & Porcentaje \\
\hline Normal & 36 & 48,6 \\
Hipoacusia ligera & 27 & 36,5 \\
Hipoacusia leve & 11 & 14,9 \\
Total & 74 & 100,0 \\
\hline
\end{tabular}

Fuente: Elaboración propia.

Se encontró que el mayor porcentaje $(48,6 \%)$ de los estudiantes de odontología presentó niveles de audiometría normal; mientras que el menor porcentaje $(14,9 \%)$ tuvo una hipoacusia leve. Además, el $36,5 \%$ presentó hipoacusia ligera. Los resultados indican que el desempeño auditivo en nuestras unidades de estudio puede conllevar a un aceleramiento de la hipoacusia presente a larga data. 


\section{TABLA No 3}

Distribución de frecuencias de la edad y la audiometría en los estudiantes de la Escuela de Odontología de la UNJBG, Tacna - 2016.

\begin{tabular}{lrrrrr} 
& \multicolumn{5}{c}{ Edad en años } \\
\cline { 3 - 6 } Audiometría & $17-20$ & $21-24$ & 25 a más & Total \\
& Normal & 21 & 13 & 2 & 36 \\
& $\%$ & 58,3 & 36,1 & 5,6 & 100 \\
Hipoacusia & $\mathrm{N}$ & 11 & 13 & 3 & 27 \\
ligera & $\%$ & 40,7 & 48,1 & 11,1 & 100 \\
Hipoacusia leve & $\mathrm{N}$ & 0 & 8 & 3 & 11 \\
& $\%$ & 0,0 & 72,7 & 27,3 & 100 \\
Total & $\mathrm{N}$ & 32 & 34 & 8 & 74 \\
& $\%$ & 43,2 & 45,9 & 10,8 & 100 \\
$\mathrm{p}=0,012<\alpha=0.05$ & entonces no se rechaza $\mathrm{H}_{\circ}$ &
\end{tabular}

Fuente: Elaboración propia.

Se evidencia que la audiometría fue normal entre las edades de 17 a 20 años obteniendo el mayor porcentaje $(58,3 \%)$, mientras que las edades que fluctúan de 21 a 24 años presentaron hipoacusia ligera con un porcentaje de $48,1 \%$ y una hipoacusia leve con un porcentaje de $72,7 \%$; es decir, a mayor edad va desmejorando el desempeño auditivo. Además, según la prueba del chi cuadrado existe relación significativa entre la edad y la audiometría en los estudiantes de la escuela de odontología de la UNJBG.

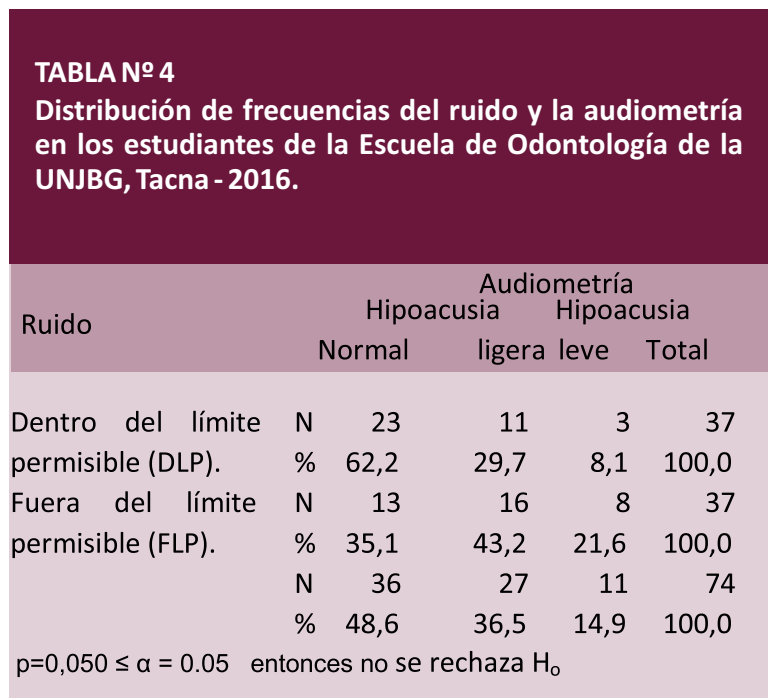

Se puede visualizar que la distribución de los porcentajes en referencia al ruido y audiometría en los estudiantes de la escuela de odontología, donde el $62 \%$ presentó audiometría normal dentro del límite permisible y un 43,2\% tuvo hipoacusia ligera fuera del límite permisible.

Según la prueba del chi cuadrado se comprobó que existe relación significativa entre los niveles de audiometría y ruido ocupacional en los estudiantes de la escuela de odontología de la UNJBG.

\section{DISCUSIÓN}

En nuestra investigación encontramos que el 50\% de los estudiantes de odontología, se encontró inmerso en un nivel de ruido DLP en los primeros tres años de estudios y el otro $50 \%$ de los estudiantes, se encontró en un nivel de ruido fuera del límite permisible, en el cuarto y quinto año de estudios. Esto se debe a que los estudiantes van aumentando las horas de exposición al ruido durante las prácticas clínicas en su formación profesional. De la misma manera, un estudio realizado en Cartagena de Indias demostró que la disminución de los niveles audiométricos de los oídos derecho e izquierdo de los estudiantes se encuentran en directa relación con la edad, el tiempo expuesto y el lugar donde estudian o trabajan (17). La valoración de ruido ambiental reportó picos de $64-94 \mathrm{~dB}$ considerados niveles relativamente elevados, fuera del límite permisible. Datos importantes; ya que en el estudio realizado por Castro Espinoza en el 2014 (18), se halló que la valoración de los niveles de ruido en las clínicas odontológicas se encontraban con picos entre 79 a 84 $\mathrm{dB}$, los cuales se consideran niveles por encima de los valores tolerables. De igual forma Urbina en el 2014 (19) demostró que el ruido en el ambiente laboral superó los 85 dB en una gran mayoría de las mediciones. Sin embargo, otros estudios contradicen nuestros resultados como el realizado por Lourenco y col. en el 2011 (20). Los autores desarrollaron un estudio cuyo objetivo fue medir las intensidades de ruido emitidas por los motores de alta rotación. Se concluyó que las intensidades de ruido emitidas por los motores de alta rotación estuvieron por debajo de límite permisible para la salud auditiva.

Además, hallamos que el $48.6 \%$ de los estudiantes de odontología, presentaba la condición auditiva normal; mientras que un $36,5 \%$ de los estudiantes se encontraba con hipoacusia ligera y un $14.9 \%$ con hipoacusia leve, rango que va haciéndose evidente tras los años transcurridos de estudio en relación con la edad del estudiante.

Otro estudio realizado por Espinoza y cols. evaluaron a 63 estudiantes de odontología y 
odontólogos, mediante audiometrías tonal clásica y de alta frecuencia, otoemisiones acústicas. En el estudio se observó que los estudiantes y odontólogos con más años de exposición a ruido odontológico presentaban peor desempeño en las pruebas de otoemisiones acústicas y se constató que los niveles de ruido eran similares en toda la muestra. Finalmente, se concluyó que no existe correlación entre niveles de ruido y pérdida auditiva; sin embargo, a mayor tiempo de exposición, menor es la amplitud de la frecuencia 4000 en otoemisiones acústicas.

Es importante señalar que la relación causa efecto es evidente, pues los estudiantes de odontología están expuestos a ruidos intensos dentro de la práctica preclínica y clínica. Los instrumentos como la turbina de alta velocidad, recortadora de modelos, la jeringa triple y el ultrasonido son los más utilizados. Gran parte de ellos funcionan a elevadas frecuencias, los cuales pueden considerarse perjudiciales para el sentido de la audición.

Finalmente, concluimos que el $50 \%$ de ruido ocupacional DLP se encuentra registrado en ambientes del primer, segundo y tercer año de la escuela de Odontología; mientras que el otro $50 \%$ de ruido ocupacional FLP se encuentra en los ambientes de cuarto y quinto año de Odontología. El 48,6\% de los estudiantes presentó condiciones acústicas normales, mientras que el $36.5 \%$ tuvo hipoacusia ligera y el $14,9 \%$ presentó hipoacusia leve. Además, se encontró que el $62 \%$ tienen audiometría normal y están DLP, mientras que el 43,2\% presentó hipoacusia ligera y están FLP. Por lo tanto, según las pruebas estadísticas sí existe relación significativa entre los niveles de audiometría y ruido ocupacional en los estudiantes de la escuela de odontología de la UNJBG en el 2016.

Se recomienda tanto el mantenimiento regular de las unidades dentales como el uso de equipos modernos menos ruidosos con el fin de disminuir el potencial de riesgo de enfermedades ocupacionales. Asimismo, es importante el acondicionamiento acústico de las áreas clínicas y preclínicas, que permitan prácticas saludables y ergonómicas que garanticen la bioseguridad de los estudiantes y docentes de éstas áreas. Adicionalmente, es necesario tomar medidas preventivas como el uso de tapones auditivos obligatorios en estudiantes y docentes de la Escuela Profesional de Odontología y establecer protocolos de prevención, indicando como controlar los riesgos que están expuestos los estudiantes de odontología y poder prevenir problemas acústicos posteriores.

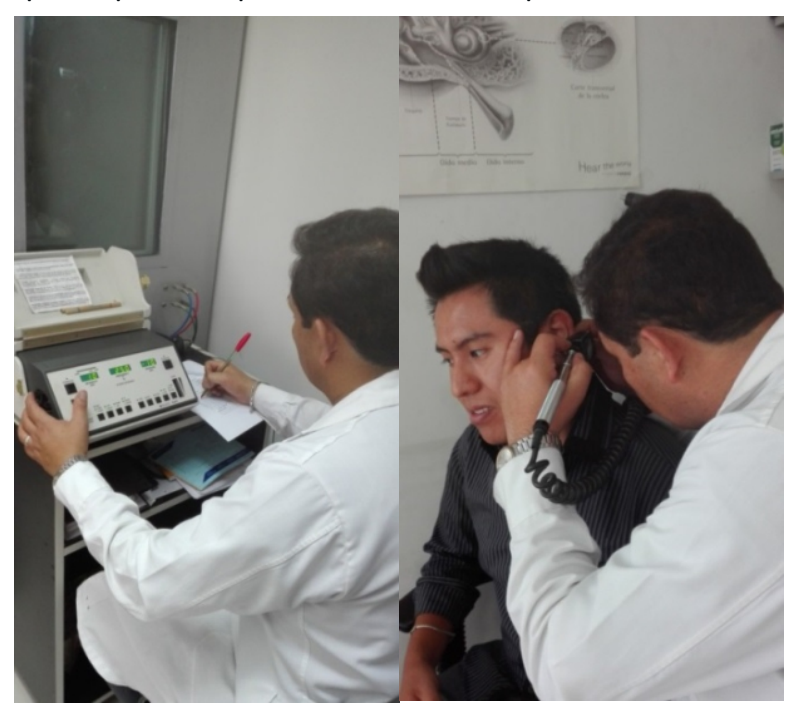




\section{REFERENCIAS BIBLIOGRÁFICA}

1. Párraga Velásquez MdR, García Zapata T. El ruido y el diseño de un ambiente acústico. Industrial Data. 2005;8(2):0.

2. al C-BMe. Occupational Noise: Assessing the burden of disease from work-related hearing impairment at national and local levels.Geneva, . World Health Organization. 2004;9.

3. Chowanadisai S, Kukiattrakoon B, Yapong B, Kedjarune U, Leggat PA. Occupational health problems of dentists in southern Thailand. International dental journal. 2000;50(1):36-40.

4. Sliwinska-Kowalska M, Davis A. Noise-induced hearing loss. Noise and Health. 2012;14(61):274-80.

5. Segués F. Conceptos básicos de ruido ambiental. Ministerio de Medio Ambiente; 2007.

6. Pujana Jyc. Medición del ruido generado en el ejercicio de la odontología en las clínicas de enseñanza de la FES Iztacala. . Revista Odontología Actual. 2007;5(56).

7. Morata TC, Fiorini AC, Fischer FM, Colacioppo S, Wallingford $\mathrm{KM}$, Krieg EF, et al. Toluene-induced hearing loss among rotogravure printing workers. Scandinavian Journal of Work, Environment \& Health. 1997(4):289-98.

8. Sampaio Fernandes JC, Carvalho APO, Gallas M, Vaz P, Matos PA. Noise levels in dental schools. European Journal of Dental Education. 2006;10(1):32-7.

9. Brusis T, Hilger R, Niggeloh R, Huedepohl J, Thiesen KW. [Are professional dental health care workers (dentists, dental technicians, assistants) in danger of noise induced hearing loss?]. Laryngo-rhino- otologie. 2008;87(5):335-40.

10. Forman-Franco B, Abramson AL, Stein T. High-speed drill noise and hearing: audiometric survey of 70 dentists. Journal of the American Dental Association (1939). 1978;97(3):479-82.

11. Chile MdS. Protocolo de Exposicion Ocupacional Ruido. 2013:14.
12. Obando Soto M, Castañeda J, Rodríguez Y, Triana C. Comportamiento Auditivo en Odontologos y Auxiliares de Odontologia que hacen uso de la Pieza de Mano como Herramienta de Trabajo (Estudio descriptivo). Umbral Científico. 2009(14):27-47.

13. Fuentes $L E$, Rubio M C, Cardemil M F. Pérdida auditiva inducida por ruido en estudiantes de la carrera de odontología. Revista de otorrinolaringología y cirugía de cabeza y cuello. 2013;73:249-56.

14. Grass Martínez Y, Castañeda Deroncelé $M$, Pérez Sánchez G, Rosell Valdenebro L, Roca Serra L. El ruido en el ambiente laboral estomatológico. MEDISAN. 2017;21:527-33.

15. Salcedo GMP. Ruido ocupacional y niveles de audiometría en el personal odonotlógico del servicio de Estomatología del Centro Naval Cirujano Mayor Santiago Távara ,Lima 2013. Tesis UNMSM Perù. 2013:4-107.

16. Díaz de León-Morales F-HOC. Daño por Exposición a Ruido Ambiental en Estudiantes de Odontología. Revista Ciencia y Trabajo. 2012;14(44):175-9.

17. Martínez FDG. Niveles Audiométricos de los estudiantes de Odontología de la Universidad de Cartagena 2013. Tesis Universidad de Cartagena Colombia. 2013:8-78.

18. Castro Espinosa J, Ortiz Julio S, Tamayo Cabeza G, González Martínez F. Niveles de Ruido en Clínicas Odontológicas de la Universidad de Cartagena. 2016. 2016;6(17):8.

19. Espinoza JOU. Evaluación y comparación del nivel auditivo de los alumnos de ll y $V$ año de la carrera de Odontología

Facultad de Ciencias Médicas UNAN-Managua en el periodo abril-septiembre 2014 Tesis de UNAM-Managua. 2014:90.

20. Lourenco Eea. Can noise in dental clinic produce hearing loss? Arquivos Internacionais de Otorrinolaringologia. 2011;15(1):84-8.

21. cols. EYy. Niveles de ruido ocupacional y desempeño audiológico en estudiantes y profesionales de Odontologìa. Tesis de UChile. 2013:4-60

Fecha de recepción: 15 de junio de 2018

Fecha de aceptación: 05 de noviembre de 2018

\section{Correspondencia}

Noelia Yesica Martínez Cántaro

E-mail: milsonrisas1000@hotmail.com 